\title{
Cold and Dense Perturbative Yukawa Theory with Massive Fermions
}

\author{
L. F. Palhares and E. S. Fraga \\ Instituto de Física, Universidade Federal do Rio de Janeiro \\ C.P. 68528, Rio de Janeiro, RJ 21941-972, Brazil
}

Received on 29 September, 2006

\begin{abstract}
Recent results for the two-loop thermodynamic potential of QCD at finite density have shown that nonzero quark mass corrections to the pressure are relevant and can dramatically affect the structure of compact stars. Motivated by these findings, we consider a simple toy model - cold and dense Yukawa theory - to study the effects of finite fermion masses on the pressure. The role of renormalization group running of the coupling and mass is also discussed. Results within this simple model might be useful in the description of condensates in the core of neutron stars.
\end{abstract}

Keywords: Finite-temperature field theory; Yukawa theory; Equation of state

The role of finite quark masses in QCD thermodynamics has received increasing attention in the last few years. Since their effects might be relevant in the description of phenomena associated with the chiral and the deconfinement transitions, more realistic investigations of the phase structure of strong interactions should incorporate them.

For finite temperature and vanishing chemical potential, lattice simulations can now investigate the effect of nonzero quark masses more reliably, and preliminary results for the corrections to the behavior of the quark condensate and the renormalized Polyakov loop with temperature have been obtained [1]. Although none of these quantities is a true order parameter under such conditions, they still provide useful information on chiral symmetry breakdown and confinement within the framework of effective field theories [2].

In the case of cold and dense QCD, it was generally believed that the effects of nonzero quark masses on the equation of state were negligible, thereby yielding only minor corrections to the mass-radius diagram of compact stars. At the time of the first investigations of (strange) quark star structure [3], the seminal computations of the thermodynamic potential for cold QCD at finite chemical potential in perturbation theory to $\sim \alpha_{s}^{2}$ had already been done [4-6]. Nevertheless, properties of quark stars were then computed making use of the bag model description of the equation of state with corrections only up to order $\alpha_{s}$ from perturbative QCD, a practice that remains in most treatments to date [7]. In the massless case, however, first-order corrections cancel out in the equation of state, so that one ends up with a free gas of quarks modified only by a bag constant. Including a finite quark mass for the strange quark, for instance, brings a correction that was first estimated to modify the equation of state by less than $5 \%$ and was essentially ignored.

In fact, mass, as well as color superconductivity gap, contributions to the pressure are supressed by two powers of the chemical potential as compared to zero-mass interacting quark gas terms. Therefore, assuming a critical chemical potential for the chiral transition of the order of a few hundred $\mathrm{MeV}$, naively those terms should not matter. However, recent results for the thermodynamic potential to two loops have shown that corrections are sizable, and may dramatically affect the structure of compact stars [8]. Moreover, the situation in which mass (as well as gap) effects are significant corresponds to the critical region for chiral symmetry breakdown in the phase diagram of QCD. Hence, not only the value of the critical chemical potential will be affected, but also the nature of the chiral transition. In particular, if the latter is strongly first-order there might be a new class of compact stars, smaller and denser, with a deconfined quark matter core [9]. Of course, contributions due to color superconductivity [10] as well as chiral condensation [11] will also affect this picture.

In this paper, we consider a simple toy model - cold and dense Yukawa theory - to study the influence of fermion masses on the pressure. The extremely simplified formal structure allows for a detailed investigation of the features that come about in higher-order contributions to the equation of state, some of which will be preserved in the case of cold and dense full QCD. Here, we present a two-loop calculation of the pressure with massive fermions in the modified minimal subtraction $(\overline{M S})$ renormalization scheme, and discuss qualitatively the role to be played by the renormalization group running of the coupling and the fermion mass. Higher-order corrections and a thorough analysis of renormalization group effects will be presented elsewhere [12].

The use of corrections $\sim \alpha_{s}^{2}$ with a modern definition of the running coupling constant to model the non-ideality in the equation of state for cold, dense QCD with massless quarks [9] has shown that the renormalization group flow plays a key role, a feature that is probably even more important in the case of massive quarks [8]. In fact, as will be discussed below, the introduction of a nonzero mass for the fermions brings the first logarithmic contribution to the equation of state to two-loop order, whereas in the massless case such corrections appear only at three loops.

Although our framework is assumedly very simplified, our results could be useful not only to provide insight for inmedium QCD phenomena, but also in many different applications, since Yukawa-type couplings appear in several contexts as will be discussed later.

In what follows, we consider a gas of massive fermions whose interaction is mediated by a real scalar field, $\phi$, with an interaction Lagrangian of the Yukawa form:

$$
\mathcal{L}_{I}=g \bar{\psi} \psi \phi
$$


where $g$ is the coupling constant.

Although we are interested here in the limit in which the temperature vanishes, it is convenient to work initially with finite temperature and density. Within the imaginary-time framework [13], the time dimension is compactified and associated with temperature $(T)$. Furthermore, imposition of periodicity or anti-periodicity of the fields in the imaginary time reflects the spin-statistics theorem, characterizing, respectively, bosons $(B)$ or fermions $(F)$. As a consequence, only specific discrete Fourier modes depending on the statistical nature of the field are allowed. Therefore, as is customary in finite-temperature field theory, integrals over the zeroth four-momentum component are mapped into discrete sums over Matsubara frequencies, denoted by $\omega_{n}^{B}=2 n \pi T$ and $\omega_{n}^{F}=(2 n+1) \pi T$, with $n$ integer. To take finite density effects into account, the chemical potential $\mu$ is included, representing, in practice, a shift in the zeroth component of the fermionic four-momentum.

From the partition function written in terms of the euclidean action $S_{E}, Z(T, \mu)=\operatorname{Tr} \exp \left(-S_{E}\right)$, one derives the perturbative series for the pressure $P$ :

$$
P=-\frac{T}{V} \ln Z=-\frac{T}{V} \ln Z_{0}-\frac{T}{V} \ln \left[1+\sum_{l=1}^{\infty} \frac{(-1)^{l}}{l !}\left\langle S_{I}^{l}\right\rangle_{0}\right]
$$

where $V$ is the volume of the system, $Z_{0}$ is the partition function of the free theory and $S_{I}$ represents the interaction action. In our case, Wick's theorem implies that only even powers in the above expansion survive. Therefore, the perturbative method results in a power series of $\alpha_{Y} \equiv g^{2} / 4 \pi$ [15]. Up to $O\left(\alpha_{Y}\right)$, the first non-trivial contributions to the pressure are given by the free massive gas term, $P_{0}$, and the "exchange diagram", $P_{1}$.

Using the Feynman rules in the imaginary time formalism at finite temperature [13], one can compute the pressure of a gas of interacting massive fermions order-by-order in perturbation theory. The free gas contribution for fermions of mass $m$ has the form:

$$
\begin{aligned}
\lim _{T \rightarrow 0} P_{0} & =\lim _{T \rightarrow 0} \sum_{P} \operatorname{Tr} \ln [\not P-m]= \\
& =\frac{1}{12 \pi^{2}}\left[\mu p_{f}\left(\mu^{2}-\frac{5}{2} m^{2}\right)+\frac{3}{2} m^{4} \ln \left(\frac{\mu+p_{f}}{m}\right)\right]
\end{aligned}
$$

where the trace is performed over the Dirac structure, $P^{\mu}=$ $\left(i \omega_{n}^{F}+\mu, \mathbf{p}\right)$ is the fermionic four-momentum, $p_{f}=\sqrt{\mu^{2}-m^{2}}$ is the Fermi momentum, and we use the following notation for the sum-integrals:

$$
\mathcal{f}=T \sum_{n} \int \frac{d^{3} \mathbf{p}}{(2 \pi)^{3}}
$$

The $O\left(\alpha_{Y}\right)$ correction is given by the exchange term [13, 14]:

$$
P_{1}=-2 \pi \alpha_{Y} \oiint_{P, Q, K} \operatorname{Tr}\left[\frac{(2 \pi)^{3} \beta \delta^{(4)}(K-P+Q)}{(P-m) K^{2}(Q-m)}\right],
$$

where $\beta=1 / T$ and $K^{\mu}=\left(i \omega_{l}^{B}, \mathbf{k}\right)$ is the bosonic fourmomentum.
Resorting to a particular representation of the Dirac delta function [14], one can rewrite the Matsubara sums in (5) in a decoupled form and solve them independently via equivalent contour integrations. We obtain, performing all the sumintegrals explicitly, the following renormalized result in the $\operatorname{limit} T \rightarrow 0$ :

$$
\begin{aligned}
\lim _{T \rightarrow 0} P_{1} & =-\frac{\alpha_{Y}}{4 \pi^{3}}\left\{\frac{3}{4}\left[\mu p_{f}-m^{2} \ln \left(\frac{\mu+p_{f}}{m}\right)\right]^{2}-p_{f}^{4}\right. \\
& \left.+m^{2}\left[3+2 \ln \frac{\Lambda^{2}}{m^{2}}\right]\left[\mu p_{f}-m^{2} \ln \left(\frac{\mu+p_{f}}{m}\right)\right]\right\},
\end{aligned}
$$

where $\Lambda$ is the renormalization scale in the $\overline{M S}$ scheme.

It is interesting to notice that the inclusion of mass for the fermions brings the presence of logarithmic corrections one order down in $\alpha_{Y}$. In massless QCD, for instance, one has $\sim \alpha_{s}$ corrections at two loops and $\sim\left\{\alpha_{s}^{2}, \alpha_{s}^{2} \ln \alpha_{s}, \alpha_{s}^{2} \ln (\Lambda / \mu)\right\}$ at three loops [9]. However, in the massive case one finds not only $\sim \alpha_{s}$ terms, but also a contribution $\sim m^{2} \alpha_{s} \ln (\Lambda / \mu)$ at two loops, as can be seen above. In our case, this feature will be important when we incorporate renormalization group running of $\alpha_{Y}$ and $m$.

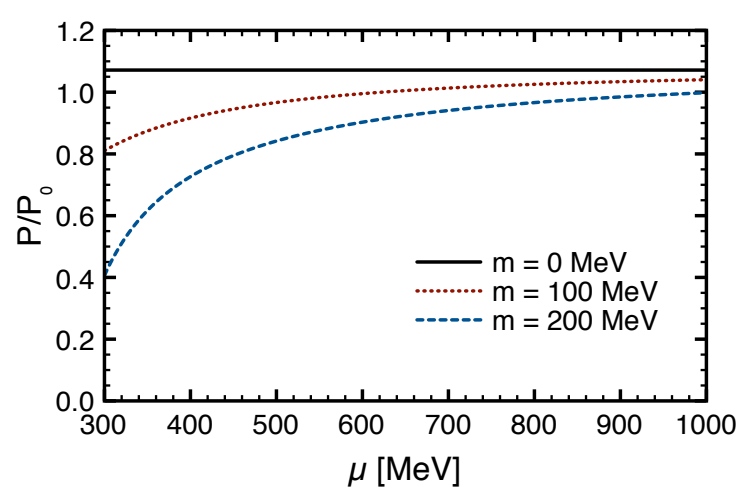

FIG. 1: Pressure normalized by the free fermion gas pressure as a function of the fermion chemical potential for $\Lambda=2 \mu$ and different values of the fermion mass.

In Fig. 1 we illustrate the effect of modifying the mass on the total pressure to $O\left(\alpha_{Y}\right), P=P_{0}+P_{1}$, normalized by the pressure of the free gas, as a function of the fermionic chemical potential. The choice of range for $\mu$, and accordingly for the masses, are inspired by the scales found in the case of QCD [8]. In the same vein, the coupling is fixed to $\alpha_{Y}=0.3$. It is clear from the figure that mass corrections bring significant changes to the pressure, even in the absence of renormalization group (RG) running for the coupling and the mass. On the other hand, to perform a RG analysis it is more convenient to plot $P$ vs. $\mu$ and look at the small $\mu$ region [12], given the sign of the beta function for this theory.

Fig. 2 displays the dependence on the renormalization scale $\Lambda$. The values chosen are motivated by the ones which appear 


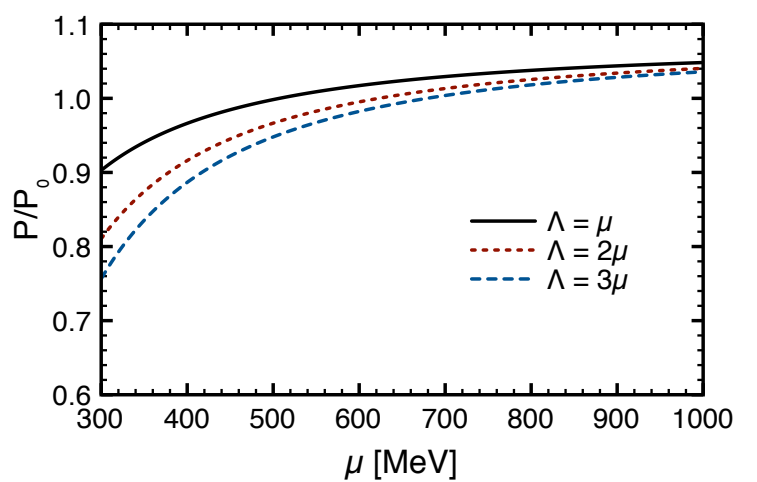

FIG. 2: Pressure normalized by the free fermion gas pressure as a function of the fermion chemical potential for $m=100 \mathrm{MeV}$ and different values of the renormalization scale $\Lambda$.

in QCD, as before. Although the effects of varying $\Lambda$ appear to be relatively small, it would be premature to conclude that this feature will remain after implementing the RG flow. In fact, the results presented in Fig. 2 most probably underestimate the scale dependence of the full correction, since not only the coupling but also the mass will run with $\Lambda$. In the Yukawa theory, in contrast to QCD, the effect will become larger as we increase the chemical potential. For fixed coupling, larger values of $\Lambda$ yield larger modifications in the pressure. However, after the inclusion of RG running, this behavior can be mantained, as should be the case here, or become the opposite, as is the case in QCD, depending on the sign of the beta function. Since the $\Lambda$-dependence comes from the term $\sim m^{2} \alpha_{Y} \ln (\Lambda / m)$ in (6), there will be a competition between the behavior of the renormalization scale $\Lambda$ and that of $m$ and $\alpha_{Y}$ as functions of $\mu$.

Recently, finite fermion mass corrections were shown to affect significantly the cold and dense equation of state for QCD [8], despite the previous disbelief in its relevance. In this work, we investigated further these effects in the context of the Yukawa theory. The first non-trivial correction to the pressure of a free gas of massive fermions at finite density was calculated. Even at two-loop order, as discussed before, mass effects bring into play logarithmic corrections originated in the $\overline{M S}$ subtraction scheme. As usual, they bring about a nonphysical dependence on the renormalization scale $\Lambda$, since one has to cut the perturbative series at some order. Higher-order computations in this framework are in progress [12], and will give a better handle on the choice of this scale, which in our case should be a function of $\mu$ and $m$. On the other hand, one can also choose the scale in a phenomenological way in a given model, imposing physical constraints to the equation of state, as was done in Ref. [9] to model the non-ideality of QCD at finite density with massless quarks.

A detailed renormalization group flux analysis is an essential step towards understanding the role of fermion masses in the equation of state at different energy scales and is also under current development [12]. These results may show that the modifications remain stable through the perturbative expansion and contribute to establish the importance of nonzero fermion mass corrections in high-density strongly interacting media. In the framework of effective theories, in which Yukawa-type couplings appear frequently, corrections due to finite fermion masses might yield appreciable deviations from the conventional results and affect different physical systems. If one also considers temperature effects, several applications are found from the realms of the electroweak phase transition in the early universe to the formation of condensates in the core of neutron stars.

\section{Acknowledgments}

The authors would like to thank R.D. Pisarski, J. SchaffnerBielich and C. Villavicencio for fruitful discussions. This work was partially supported by CAPES, CNPq, FAPERJ and FUJB/UFRJ.
[1] C. Bernard et al. [MILC Collaboration], Phys. Rev. D 71, 034504 (2005); Z. Fodor and S. D. Katz, JHEP 0404, 050 (2004); P. Petreczky and K. Petrov, Phys. Rev. D 70, 054503 (2004); Y. Aoki, Z. Fodor, S. D. Katz, and K. K. Szabo, JHEP 0601, 089 (2006); P. de Forcrand and O. Philipsen, heplat/0607017.

[2] A. Dumitru, D. Roder, and J. Ruppert, Phys. Rev. D 70, 074001 (2004); A. Mocsy, J. Phys. G 31, S1203 (2005); J. Braun, B. Klein, H. J. Pirner, and A. H. Rezaeian, Phys. Rev. D 73, 074010 (2006).

[3] E. Witten, Phys. Rev. D 30, 272 (1984); E. Farhi and R. L. Jaffe, Phys. Rev. D 30, 2379 (1984); C. Alcock, E. Farhi, and A. Olinto, Astrophys. J. 310, 261 (1986); P. Haensel, J. L. Zdunik, and R. Schaeffer, Astron. Astrophys. 160, 121 (1986).

[4] B. A. Freedman and L. D. McLerran, Phys. Rev. D 16, 1130 (1977); ibid., D 16, 1147 (1977); ibid., D16, 1169 (1977); ibid.,
D 17, 1109 (1978).

[5] V. Baluni, Phys. Rev. D 17, 2092 (1978).

[6] T. Toimela, Int. J. Theor. Phys. 24, 901 (1985) [Erratum-ibid. 26, 1021 (1987)].

[7] N. K. Glendenning, Compact Stars - Nuclear Physics, Particle Physics, and General Relativity (Springer, New York, 2000); H. Heiselberg and M. Hjorth-Jensen, Phys. Rept. 328, 237 (2000).

[8] E. S. Fraga and P. Romatschke, Phys. Rev. D 71, 105014 (2005); E. S. Fraga, Nucl. Phys. A 774, 819 (2006).

[9] E. S. Fraga, R. D. Pisarski, and J. Schaffner-Bielich, Phys. Rev. D 63, 121702 (2001); E. S. Fraga, R. D. Pisarski, and J. Schaffner-Bielich, Nucl. Phys. A 702, 217 (2002).

[10] M. Alford and S. Reddy, Phys. Rev. D 67, 074024 (2003); M. Alford, M. Braby, M. W. Paris, and S. Reddy, Astrophys. J. 629, 969 (2005). 
[11] M. Buballa and M. Oertel, Nucl. Phys. A 703, 770 (2002); S. B. Ruster, V. Werth, M. Buballa, I. A. Shovkovy, and D. H. Rischke, Phys. Rev. D 72, 034004 (2005); D. Blaschke, S. Fredriksson, H. Grigorian, A. M. Oztas, and F. Sandin, Phys. Rev. D 72, 065020 (2005).

[12] L. F. Palhares and E. S. Fraga, work in progress.

[13] J. I. Kapusta, Finite-temperature field theory (Cambridge Uni- versity Press, 1989).

[14] J. I. Kapusta, Nucl. Phys. B 148, 461 (1979).

[15] Since we are concerned only with the zero-temperature limit, there are no odd powers of $g$ coming from resummed contributions of the zero Matsubara mode for bosons in the perturbative series. 